\title{
THE DIGITAL IMAGE EXPERIMENT PLATFORM BASED ON EVEN LINER GRAMMAR
}

\author{
Limin $\mathrm{Ao}^{1,2} \mathrm{Bo} \mathrm{Li}^{2}$ Yongsheng Chen ${ }^{2}$ \\ 1. College of Information and Electrical Engineering, China Agricultural University, Beijing \\ 100083, China \\ 2. Department of Information Engineering, Northeast China Institute of Electric Power \\ Engineering, Jilin 132012, China
}

\begin{abstract}
This paper describes a digital image processing experiment platform which is on the theory basis of the Even Linear Grammar. The platform is based on the Automatization of COM, which can increase the reusability of the image processing methods. In the image processing process, an Image Processing Process Tree (IPPT) is generated, which can make the image analysis easy.
\end{abstract}

Key words: $\quad$ syntax-directed, COM, IPPT, Reusable, Dynamically Construct

\section{INTRODUCTION}

In the process of image processing, in order to achieve our goal, we have to do many experiments and research, even to modify or design arithmetic to meet our need. In existing image processing software, Photoshop can't modify or add arithmetic; Matlab can do this, but everything must be preestablished, it is impossible to add some arithmetic into a program dynamically, and it can't record the middle results.

This paper is trying to describe an image processing platform which is based on constructed language and Syntax-directed Method.

Even Liner Grammar ${ }^{[1]}$ is used to describe a constructed language. We can program using constructed language.

In general, an image-processing program is composed of some common image-processing methods. We can make use of the structure of attribute 
grammar to reconstruct the image processing methods to be semantic functions of attribute grammar.

One of the ways to increase the reusability of the image processing method is to use COM.

$\mathrm{COM}^{[2]}$ is short for Component Object Model. COM allows the COM components communicate alternately in a uniform way.

\section{EVEN LINER GRAMMAR AND SYNTAX- DIRECTED METHOD}

Let $\mathrm{F}$ be an alphabet. A ELG(Even Liner Grammar) whose form is $\mathrm{G}=(\mathrm{N}, \Sigma, \mathrm{P}, \mathrm{X})$ is a context-free grammar. Its productions have the form like:

$$
\mathrm{A} \rightarrow \mathrm{uBv} \text { or } \mathrm{A} \rightarrow \mathrm{w}
$$

Thereinto, $A, B \in N ; u, v, w \in \Sigma^{*} ;|u|=|v|$. Grammar $G$ is a linear grammar. $\mathrm{N}$ is a non-terminal symbols set, $\Sigma$ is the terminal symbols set, $\mathrm{P}$ is the set of productions and $\mathrm{X}$ is the started non-terminal symbol.

The definition of the language based on Grammar G:

$$
L=L(G)_{*}=\left\{s \mid s \in \Sigma^{*}, X \stackrel{*}{\Rightarrow} s\right\}
$$

Thereinto, the form $X \Rightarrow s$ expresses that the string s can be inferred from the started non-terminal symbol $\mathrm{X}$ by a serious of productions.

Takada expresses his viewpoint in literature ${ }^{[3]}$ : the inference of ELG can be come down to the inference of DFA(Deterministic Finite Automata).

Takada's thinking:

There is an alphabet $\mathrm{F}$ and a $\mathrm{ELG} G=(\mathrm{N}, \Sigma, \mathrm{P}, \mathrm{X})$. Sign every production in $\mathrm{P}$ with a single letter in $\mathrm{F}$. Let $\mathrm{f}=\mathrm{f}_{1} \mathrm{f}_{2} \ldots \mathrm{f}_{\mathrm{k}} \in \mathrm{F}^{*}$. the form $X \Rightarrow f_{G} \omega$ expresses that the string $\omega$ can be inferred by the productions $f_{1} f_{2} \ldots f_{k}$ from the started non-terminal symbol $X$ of $G$. Let $C$ be a language on the alphabet $F$, then the language generated by the Grammar $\mathrm{G}$ on the control set $\mathrm{C}$ can be defined as:

$$
L_{C}(G)=\left\{\omega \in \Sigma^{*} \mid X \stackrel{*}{\Rightarrow} f_{G} \omega, f \in C\right\}
$$

So every sentence in the control set $C$ corresponds to the inference process of a sentence in $L_{C}(G)$.

Takada proves that:

Any Even Liner Language $L$ can be generated by a universal ELG $G^{0}$ on a control set $\mathrm{C}$ which is a regular language. $\mathrm{L}$ and $\mathrm{G}^{0}$ are all on an alphabet $\Sigma$. That is to say, for any ELL(Even Liner Language) $\mathrm{L}$, there is a form like:

$$
L=L_{C}\left(G^{0}\right)=\left\{\omega \in \Sigma^{*} \mid X^{0} \Rightarrow f_{G^{0}} \omega, f \in C\right\}
$$

Thereinto, $\mathrm{C}$ is a regular grammar, $\mathrm{G}^{0}$ is defined as

$$
G^{0}=\left(\left\{X^{0}\right\}, \Sigma, P^{0}, X^{0}\right) \text {. }
$$




$$
\mathrm{P}^{0}=\left\{\mathrm{X}^{0} \rightarrow \mathrm{a} \mathrm{X}^{0} \mathrm{~b} \mid \mathrm{a}, \mathrm{b} \in \Sigma\right\} \cup\left\{\mathrm{X}^{0} \rightarrow \mathrm{ab} \mid \mathrm{a}, \mathrm{b} \in \Sigma\right\} \cup\left\{\mathrm{X}^{0} \rightarrow \mathrm{a} \mid \mathrm{a} \in \Sigma\right\} \cup\left\{\mathrm{X}^{0} \rightarrow \varepsilon\right\}^{[1]} .
$$

Takada's thinking is used in this experiment platform.

Syntax-directed Method is the typical method to analyze the meaning of a sentence. The Syntax-directed Method is used in this experiment platform alternately in the image processing process. The platform uses the Syntaxdirected Method to determine the style of the operation, so as to construct an Image Processing Process Tree (IPPT) dynamically. A node in IPPT is an image. If there is a path between two nodes, it means that one image is acquired form the other image by some image processing method. The methods used in the IPPT can form a program. So the program represents the image processing process.

\section{BRIEF INTRODUCTION OF COM}

What COM (Component Object Model) provides is not only the criterion for components alternation, but also the alternation environment.

a) Specialities of COM

Theoretically speaking, COM is a criterion about $\mathrm{C} / \mathrm{S}$.

COM has the specialities of Language Independence, Robust Versioning, Local Transparency and Object Orientation.

b) Automatization

Automatization is a special example of COM. It is based on COM, but its use is much wider than COM. Automatization provides normal attempering measures for the transmission of parameters and return values between different processors or different computers.

c) Later Binding

Later Binding is one of the important characteristics of Automatization. That is, the client program doesn't check the data type of the parameters when it is programmed, but check when the component is used.

\section{THE THEORY BASIS OF THE IMAGE PROCESSING PROCESS}

In the experiment platform, along with the image processing process, a image processing process tree (IPPT) can be constructed. The root of the tree is the original image, the middle nodes are the images generated in the processing process, and the branches are the processing functions used in the platform. Users of the platform can easily know how an image is generated in the platform by along the branches of IPPT. 
Let $R_{1}$ be the original image, $R_{2}, R_{3}, \ldots, R_{n}$ be the generated images in the image processing process, $f_{1}, f_{2}, \ldots, f_{n}$ be some function in the processing process. Then, there is a IPPT:

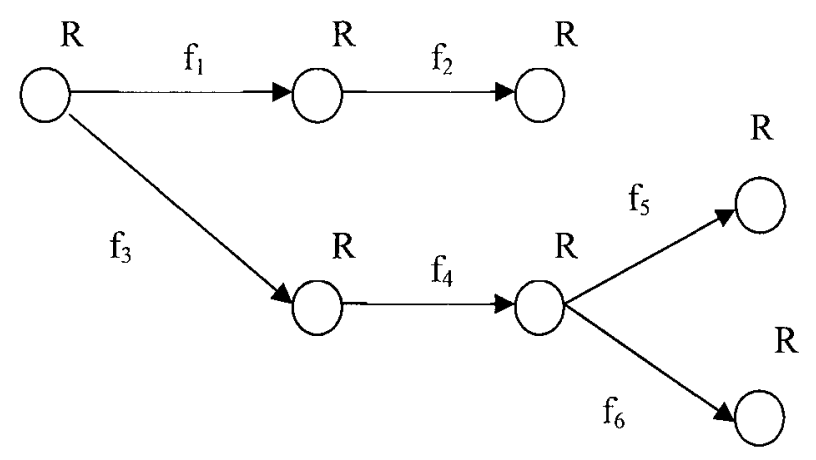

Figure 1. An IPPT

As figurel shows, the original image $\mathrm{R}_{1}$ can generate a serious of images by the image processing process. Supposing $R_{7}$ is the image that the user wants. The user can know how $\mathrm{R}_{7}$ is acquired by analyzing the IPPT. And the user can save the process of generating $R_{7}$ as one operation, so as to simplify the operation process when it is used next time.

At the same time, every image generated in the processing process can be used as a root node to generate another IPPT, and the tree can go on and on.

Formalize the image processing process:

Another image will be generated if an image is processed by a specific arithmetic. And the generated image can be processed again. Let $X, X^{\prime}, X^{\prime}$, represent an image, and $a, b, \ldots$ represent some specific image processing arithmetic, then the image processing process can be expressed as:

$$
\mathrm{X} \rightarrow \mathrm{aX}, \mathrm{X}^{\prime} \rightarrow \mathrm{bX}, \text {. }
$$

To the image experiment platform, image is just data, and an image processing arithmetic is just an operation.

So the image processing process can be described as: $X \rightarrow a X$.

Thereinto, $X$ is some image, $\alpha$ is some image processing arithmetic.

So every operation in the experiment platform can be considered as an application of regular grammar. RLG is a kind of regular grammar. Let a operation in the platform be $f_{i}$.

Thereout, we can define a regular language $\mathrm{C}$, its corresponding regular grammar is: $\mathrm{G}_{\mathrm{C}}=\left(\mathrm{N}_{\mathrm{C}}, \Sigma_{\mathrm{C}}, \mathrm{P}_{\mathrm{C}}, \mathrm{X}_{\mathrm{C}}\right)$. Thereinto, $\mathrm{N}_{\mathrm{C}}=\left\{\mathrm{X}_{\mathrm{C}}\right\}, \Sigma_{\mathrm{C}}=\left\{\mathrm{f}_{\mathrm{l}}, \mathrm{f}_{2}, \ldots, \mathrm{f}_{\mathrm{k}}\right\}$, $\mathrm{P}_{C}=\left\{\mathrm{X}_{\mathrm{C}} \rightarrow \mathrm{f}_{\mathrm{i}} \mathrm{X}_{\mathrm{C}} \mid \mathrm{f} \in \Sigma_{\mathrm{C}}\right\} \cup\left\{\mathrm{X}_{\mathrm{C}} \rightarrow \mathrm{f}_{\mathrm{i}} \mid \mathrm{f}_{\mathrm{i}} \in \Sigma_{\mathrm{C}}\right\}$

The image experiment platform provides the function to save a serious of image processing operations to be one operation. That is to say, define a new operation $f$, let $f=f_{1} f_{2} \ldots f_{k}$. if $F^{*}$ is the set of all the combinations of the 
arithmetic in the platform, then $f \in \mathrm{F}^{*}$. And $\mathrm{f}$ can be inferred in regular language $\mathrm{C}$, so $\mathrm{f} \in \mathrm{C}$.

On the other hand, the operation result can be described in 3 ways: the image data will be written into database, the DDB data to display the image in the screen, and the record about the current operation generated in the image processing process. So an operation $f_{i}$ can be described as $X \rightarrow a X^{\prime} \beta$, thereinto, $X$ is the original image, $X^{\prime}$ is the generated image, $\alpha$ is the image data written into the database, and $\beta$ is the operation record. $|\alpha|=|\beta|=1$. When we get the image we want, it's not necessary to process any more. We just need to save the image data into database and record the information about the current arithmetic. In that case, the operation can be described as

$$
X \rightarrow \alpha \beta \text { or } X \rightarrow \alpha \text {. }
$$

Thereout, we can get an ELG $G^{0}$, which is suited to this image processing experiment platform:

$$
G^{0}=\left(\left\{X^{0}\right\}, \Sigma, P^{0}, X^{0}\right)
$$

Thereinto, $\mathrm{P}^{0}=\left\{\mathrm{X}^{0} \rightarrow \alpha \mathrm{X}^{0} \beta \mid \alpha, \beta \in \Sigma\right\} \cup\left\{\mathrm{X}^{0} \rightarrow \alpha \beta \mid \alpha, \beta \in \Sigma\right\} \cup\left\{\mathrm{X}^{0} \rightarrow \alpha \mid \alpha \in \Sigma\right\}$.

According to Takada's proof, ELG $G^{0}$ can generate ELL $L_{C}\left(G^{0}\right)$ on the control set $\mathrm{C}$.

$$
L_{C}\left(G^{0}\right)=\left\{\omega \in \Sigma^{*} \mid X^{0} \Rightarrow f_{G^{0}} \omega, f \in C\right\}
$$

$\omega$ is a combination of the operation results.

Analyze the IPPT in figurel:

The production set of ELG $\mathrm{G}^{0}$ is:

$f_{1}: R \rightarrow \alpha_{1} R \beta_{1}$

$f_{2}: R \rightarrow \alpha_{2} R \beta_{2}$

$f_{3}: R \rightarrow \alpha_{3} R \beta_{3}$

$\mathrm{f}_{4}: \mathrm{R} \rightarrow \alpha_{4} \mathrm{R} \beta_{4}$

$f_{5}: R \rightarrow \alpha_{5} R \beta_{5}$

$\ldots$

$f_{n}: R \rightarrow \alpha_{n} R \beta_{n}$

If the number of image processing functions in the platform is $n$, the number of production as above is $\mathrm{n}$. In the production, the non-terminal symbol is $\mathrm{X}$. At the same time, $\mathrm{X}$ is the started non-terminal symbol.

$\alpha_{i}$ and $\beta_{i}$ are the terminal symbols in the productions. $\alpha_{i}$ represents the image data written into database, $\beta_{i}$ represents the current operation record.

We plight here, when it is not necessary for an image to be processed more, we use the production $X \rightarrow \alpha$ to acquire the image data.

So, we need to define a production $\mathrm{f}_{0}: \mathrm{R} \rightarrow \alpha$.

So in figure 1, different path represents different processing process.

The inference process of $\mathrm{R}_{3}$ :

$$
R \stackrel{f_{1}}{\Rightarrow} \alpha_{1} R \beta_{1} \stackrel{f_{2}}{\Rightarrow} \alpha_{1} \alpha_{2} R \beta_{2} \beta_{1} \stackrel{f_{0}}{\Rightarrow} \alpha_{1} \alpha_{2} \alpha \beta_{2} \beta_{1}
$$


The inference process of $\mathrm{R}_{6}$ :

$$
R \stackrel{f_{3}}{\Rightarrow} \alpha_{3} R \beta_{3} \stackrel{f_{4}}{\Rightarrow} \alpha_{3} \alpha_{4} R \beta_{4} \beta_{3} \stackrel{f_{5}}{\Rightarrow} \alpha_{3} \alpha_{4} \alpha_{5} R \beta_{5} \beta_{4} \beta_{3} \stackrel{f_{0}}{\Rightarrow} \alpha_{3} \alpha_{4} \alpha_{5} \alpha \beta_{5} \beta_{4} \beta_{3}
$$

The inference process of $R_{7}$ :

$$
R \stackrel{f_{3}}{\Rightarrow} \alpha_{3} R \beta_{3} \stackrel{f_{4}}{\Rightarrow} \alpha_{3} \alpha_{4} R \beta_{4} \beta_{3} \stackrel{f_{6}}{\Rightarrow} \alpha_{3} \alpha_{4} \alpha_{6} R \beta_{6} \beta_{4} \beta_{3} \stackrel{f_{0}}{\Rightarrow} \alpha_{3} \alpha_{4} \alpha_{6} \alpha \beta_{6} \beta_{4} \beta_{3}
$$

Supposing that $R_{7}$ is the image we want. We can know that $R_{7}$ is acquired from $R_{1}$ via the image processing arithmetic $f_{3}, f_{4}, f_{6}$. Let $f_{G}{ }^{0}=f_{3} f_{4} f_{6}$, then the inference process of $R_{7}$ can be described as:

$$
R_{1} \Rightarrow f_{G^{\circ}} R_{7}
$$

$\mathrm{f}_{\mathrm{G}}{ }^{0}$ can be inferred by the regular Grammar $\mathrm{C}$, so $\mathrm{f}_{\mathrm{G}}{ }^{0} \in \mathrm{C}$.

So it is can be considered that the inference process of $R_{7}$ is a sentence in $\mathrm{L}_{\mathrm{C}}\left(\mathrm{G}^{0}\right)$ which is generated by ELG $\mathrm{G}^{0}$ on the control set $\mathrm{C}$.

So we can say that the image experiment platform is an application of ELL $L_{C}\left(G^{0}\right)$.

\section{THE IMPLEMENT OF THE PLATFORM}

\subsection{System Framework of the Platform}

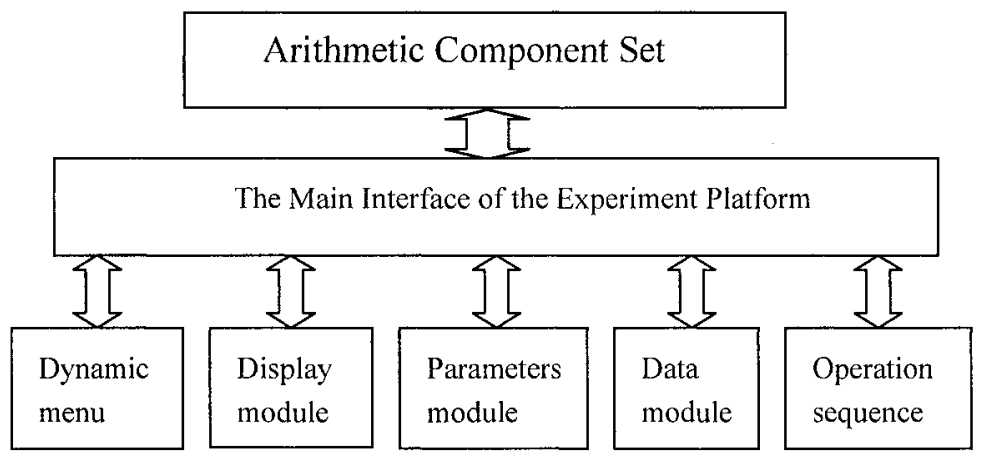

Figure 2. System Framework

The system framework is showed in figure 2. The ability of every component is:

(1) Arithmetic component set $\left(\mathrm{P}^{0}\right)$ : the set of the arithmetic about image processing including the arithmetic you programmed yourself.

(2) The dynamic menu module: implement the function to generate dynamic menus from the methods in COM components.

(3) Display module: to display the image in a new window, this is generated in the image processing process. 
(4) Parameters acquired module: to acquire the information about parameters from the database, and generate a parameter dialog dynamically which is used to implement the alternation between the user and the program.

(5) Data-saving and data-query module: to save the data acquired from the experiment process, so as to the data can be query later. In fact, the data saved is the IPPT generated in the image processing process.

(6) Save and execute the operation sequence: this module can save a serial of operations to be one operation, and then execute it.

That is equal to define a new operation $f$, and let $f=f_{1}, f_{2}, \ldots f_{n}, f_{i}$ is an operation.

\subsection{The design and implement of the interface of COM server}

In order to meet the demand of the platform, the design of the interface of the COM server must fit the condition below:

(1) The odd interface methods must have the same parameter form, and so as the even interface methods. By this way, the platform can process the method in two common ways.

(2) Every arithmetic in this platform is implemented in two interface methods. The odd method is used to retrieve the string which describes the function of arithmetic, and the even method implement the function of the arithmetic.

For example:

interface ILinear : IDispatch

\{ [id(1), helpstring("gray linear truncation")] HRESULT GetHelpString_1([out,retval]VARIANT *IResult);

[id(2), helpstring("gray linear truncation ")] HRESULT GrayLinearOff([in]unsigned char *ArrayGray, [in]long nWidth, [in]long nHeight, [in]float *pfset, [out,retval]VARIANT *IResult); $\cdots \cdots\}$

The methods id(1) and id(2) complete the "gray linear truncation" function together. The method id(2) implement the arithmetic, and the method id(1) retrieves the string "gray linear truncation" which will be used as the name of a menu item in the platform.

\subsection{The Retrospect Ability of the Platform}

The processing object of every operation in the platform is the image displayed in a child window. And the image generated by the operation will 
be displayed in a new child window. So the contrast between the two images will be much more pronounced, the original image can be reprocessed again.

In this way, every node in the IPPT generated in the image processing process can be processed as a root node.

\subsection{Save the Function Sequence}

Implement the ability like: define a new operation $f$, let $f=f_{1}, f_{2}, \ldots f_{n}$.

The platform implements the ability by record the information about every operation.

For example:

In order to find the boundary of a target area, we should operate like this:

(1) gray linear truncation, generating image 1, record as "Linear_Off 1002550255 ";

(2) finding the edge of image 1, generating image 2, record as "Sobel";

(3) turning image 2 to white-black image :image 3 , the threshold is 128. record as "Two_Value 128 ";

(4) edge trace image 3, record as "Edge Trace".

Save the operations above in a file named "Get_object_edge.serial".

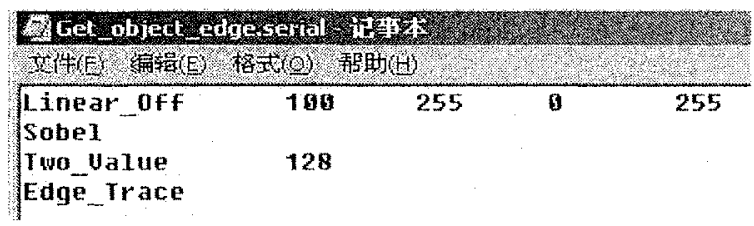

Figure 3. Get_object_edge.serial

If it is needed to perform the operation above again, the user just need to execute the Get_object_edge.serial. And the content in the file can be modified to be the appropriate content. This ability makes the operation more agile.

\section{CONLUSION}

The Image Processing Experiment Platform has powerful expansibility; it provides a reliable experiment platform for image processing.

\section{REFERENCE}

[1] ZHANG Rui-Ling. Grammatical Inference: Retrospect and Prospect. Journal of software. $1999,10(8): 850 \sim 860$

[2] Dale Rogerson. COM Technology Inside. Beijing: Publishing House of Tsinghua University, 1999 
[3] Takada Y. Grammatical inference for even linear languages based on control sets. Information Processing Letters, 1988,28(4):193 199 